\title{
Quiste dentígero asociado con mesiodens: Exposición de un caso, revisión de la literatura y diagnóstico diferencial
}

\section{Dentigerous cyst associated with mesiodens: a case report, literature review and differential diagnostic}

\author{
Rodríguez Romero FJ*, Cerviño Ferradanes S**, Muriel Cueto P***
}

\section{RESUMEN}

El quiste dentígero es uno de los quistes odontogénicos más frecuentes y están asociados con la corona de un diente no erupcionado o en desarrollo. Cuando se asocian con dientes supernumerarios son poco frecuentes y constituyen el 5-6\% de todos los quistes dentígeros. En región maxilar, el $90 \%$ de los casos se asocian a mesiodens. El objetivo de este artículo es exponer el caso de una adolescente de 14 años con un quiste dentígero asociado con un diente supernumerario (mesiodens) impactado en región maxilar anterior, su manejo dental y revisión de la literatura.

Palabras clave: Quiste dentígero, dientes supernumerarios, mesiodens.

\section{SUMMARY}

Dentigerous cyst is one of the most prevalent types of odontogenic cyst and is associated with crown of an unerupted or developing tooth. Dentigerous cysts associated with supernumerary teeth are rare and estimated to constitute $5-6 \%$ of all dentigerous cysts. The vast majority, about $90 \%$, are associated with a maxillary mesiodens. The purpose of this article to report the case of an 14-year-old a teenager with a dentigerous cyst associated with an impacted anterior maxillary supernumerary tooth, its dental management and literature review.

Key words: Dentigerous cyst, supernumerary teeth, mesiodens.

Fecha de recepción: 15 de junio de 2010.

Aceptado para publicación: 15 de julio de 2010.

* Dentista de la U.G.C. de Salud Bucodental, Distrito de Atención Primaria "Cádiz-La Janda“, Centro de Salud "La Paz". Cádiz.

** Médico Estomatólogo de la U.G.C. de Salud Bucodental, Distrito de Atención. Primaria "Cádiz-La Janda" Centro de Salud "La Laguna" Cádiz.

*** Médico Anatomopatólogo Servicio de Anatomía Patológica , Hospital Universitario Puerta del Mar" Cádiz.

Rodríguez Romero FJ, Cerviño Ferradanes S, Muriel Cueto P. Quiste dentígero asociado con mesiodens: Exposición de un caso, revisión de la literatura y diagnóstico diferencial. Av. Odontoestomatol 2011; 27 (6): 289-299. 


\section{INTRODUCCIÓN}

La clasificación de los quistes epiteliales e inflamatorios citada por Bascones (1) y propuesta por la O.M.S., en el año 1992, divide a estos quistes en:

\section{a. Quistes EPITELIALES}

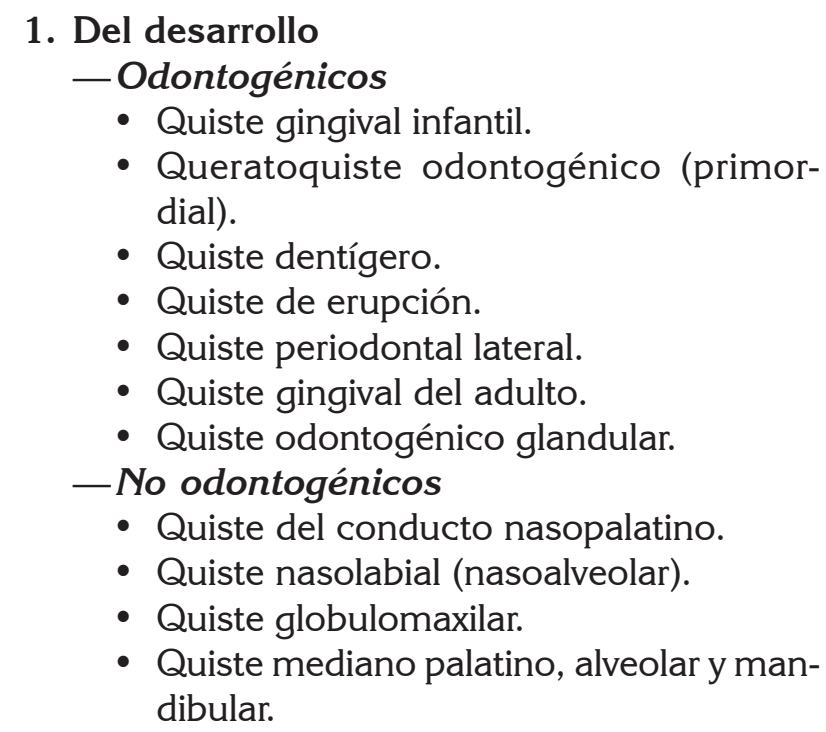

\section{Inflamatorios}

- Quiste radicular (apical, lateral o residual) —Quiste paradental (colateral inflamatorio)

\section{b. Quistes no ePITEliales}

- Quiste óseo solitario.

- Quiste óseo aneurismático.

Los quistes dentígeros se desarrollan en el órgano adamantino de un diente no erupcionado. Son por tanto quistes odontogénicos epiteliales consecutivos a alteraciones del desarrollo. (1)

En su etiología se barajan factores: genéticos, procesos degenerativos de la pulpa adamantina, acompañados con hipoplasia de esmalte y lesiones inflamatorias del germen dentario. (1)

Cuando se presentan en dentición completa erupcionada, su diagnóstico suelen ser una sorpresa y por lo general son descubiertos en exámenes radiográficos. (2)

Se desarrollan al acumularse fluido (liquido) en el espacio existente entre el epitelio reducido del es- malte y el esmalte del diente, provocando la expansión del folículo. (2)

La necrosis de la pulpa dental, es una complicación que se produce comúnmente en dientes temporales traumatizados, y esta puede ser un factor etiológico, en el desarrollo de éstos en la dentición primaria. $(3,4,9)$

Como la histopatología de su envoltura no es específica, su diagnóstico será radiológico, histológico y quirúrgico observando su unión cemento-esmalte. (5)

Aproximadamente el $95 \%$, se relacionan con dientes permanentes y solo el 5-6\% se asocian con dientes supernumerarios, como en este caso concreto. (6) La mayoría de los últimos se desarrollan en maxilar superior, alrededor de un mesiodens. $(5,6)$ y la edad habitual de presentación es en la segunda y tercera décadas. $(1,6)$ El mesiodens es el diente supernumerario más corriente en la población. (7)

Los dientes mas afectados son los $3^{\circ}$ molares mandibulares, seguidos por los caninos maxilares, terceros molares maxilares, y raras veces se asocian con incisivos maxilares y con supernumerarios. $(1,6,8)$

\section{CASO CLÍNICO}

Paciente de sexo femenino de 14 años de edad, que acude a consulta de Odontología en el Centro de Salud "La Paz", perteneciente a la Unidad de Gestión Clínica de Salud Bucodental del Distrito Sanitario Bahía de Cádiz- La Janda en la Ciudad de Cádiz. De manera fortuita y como consecuencia de un estudio radiográfico previo al tratamiento de Ortodoncia (Ortopantomografía), se descubre una imagen radiolúcida relacionada con un diente supernumerario retenido, compatible con quiste dentígero. La paciente no presenta ninguna sintomatología. Entre sus antecedentes personales y familiares encontramos, la presencia de diente supernumerario en un hermano y en su padre (mesiodens), los cuales fueron exodonciados. En la exploración extraoral presenta, el labio superior ligeramente elevado y un ligero borramiento del surco nasogeniano (Fig. 1). 


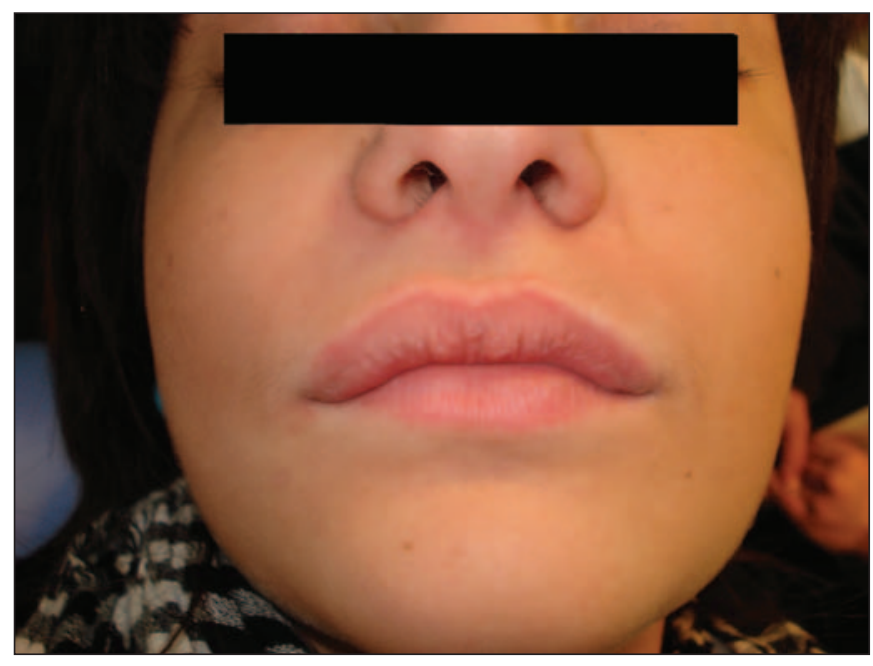

Fig. 1.

El examen intraoral, presenta crecimiento en región vestibular. La tabla externa de hemimaxila derecha a nivel de los dientes 1.1,1.2,1.3, esta bien definida, de consistencia dura, y no se aprecia crepitación, ni es pulsátil. La cortical se presenta expandida y la mucosa no presenta signos de inflamación (Fig. 2).

No se aprecian signos de afectación periodontal, no presenta lesiones por caries y nunca ha tenido molestias en la zona. En la región palatina, no presenta signos de crecimiento y la mucosa se encuentra intacta. En arcada dentaria destaca la presencia del canino deciduo 5.3 y una ligera inclinación hacia

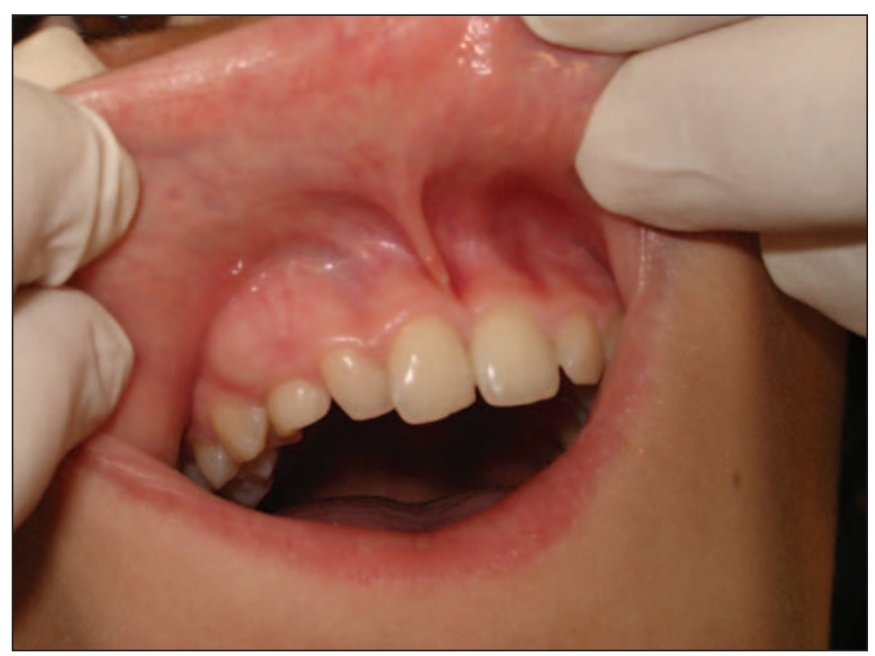

Fig. 2. mesial de las coronas del incisivo lateral 1.2 y de su ápice hacia distal. El canino 1.3 se encuentra retenido y desplazado hacia distal, y no se observa diastema entre incisivos centrales. No existe movilidad de los incisivos, aumento de la sensibilidad dentaria ni antecedentes traumáticos.

Ante la sospecha de la presencia de una lesión quística (QD) se realizó estudio radiográfico, el cual consistió en radiografías periapical, oclusal, panorámica y T.C. En la ortopantomografia (Fig. 3), se puede apreciar en hemimaxila derecha, una imagen radiolúcida, unilocular, de aproximadamente $2 \times 2 \mathrm{~cm}$, bien delimitada, asociada a un diente supernumerario (mesiodens) retenido, en posición horizontal y desplazado en dirección apical contraria a su dirección normal de erupción, directamente relacionado con la cavidad quística y la presencia de un diente supernumerario suplementario en hemimaxila izquierda.

El diente supernumerario (mesiodens) relacionado con el quiste, presenta una corona de morfología rudimentaria, con raíz corta (morfología dismórfico o conoide), sin embargo el $2^{\circ}$ supernumerario es de morfología normal, parecido a un incisivo lateral (eumórfico o suplementario) y su dirección es invertida, no presentando patología asociada. Ambos se encontraban localizados en región palatina.

En las radiografías periapical (Fig. 4) y oclusal (Fig. 5) se puede observar con más nitidez los dos super-

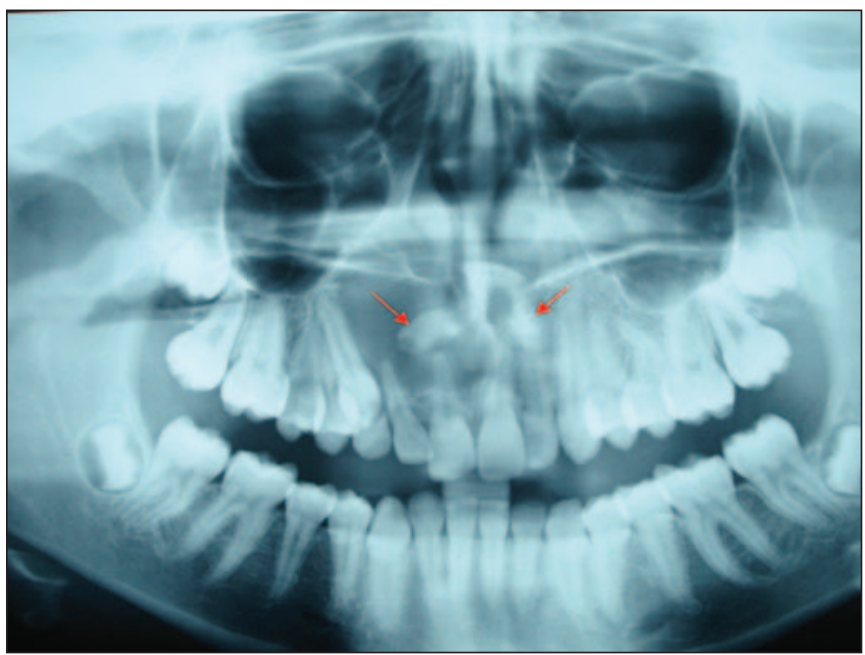

Fig. 3. Panorámica. 


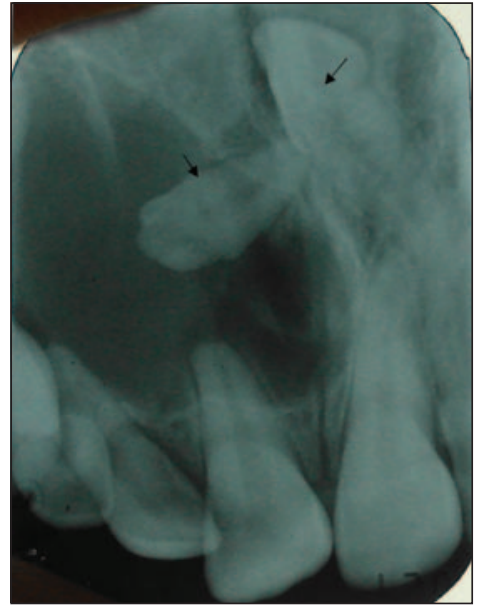

Fig. 4. Rx periapical.

numerarios, así como la lesión quística, bien delimitada y la relación de ésta con los dientes adyacentes, se puede observar que la lesión quística traspasa la línea media afectando a la hemimaxila izquierda y la intima relación del quiste con la corona del diente supernumerario. También se aprecia una ligera reabsorción de la raíz del 1,1.

Con la intención de saber mejor si la localización eran por palatino o vestibular se realizo T.C. Facial/ Senos sin contraste intravenoso, con algoritmo óseo, en plano axial y posterior reconstrucción en coronal.

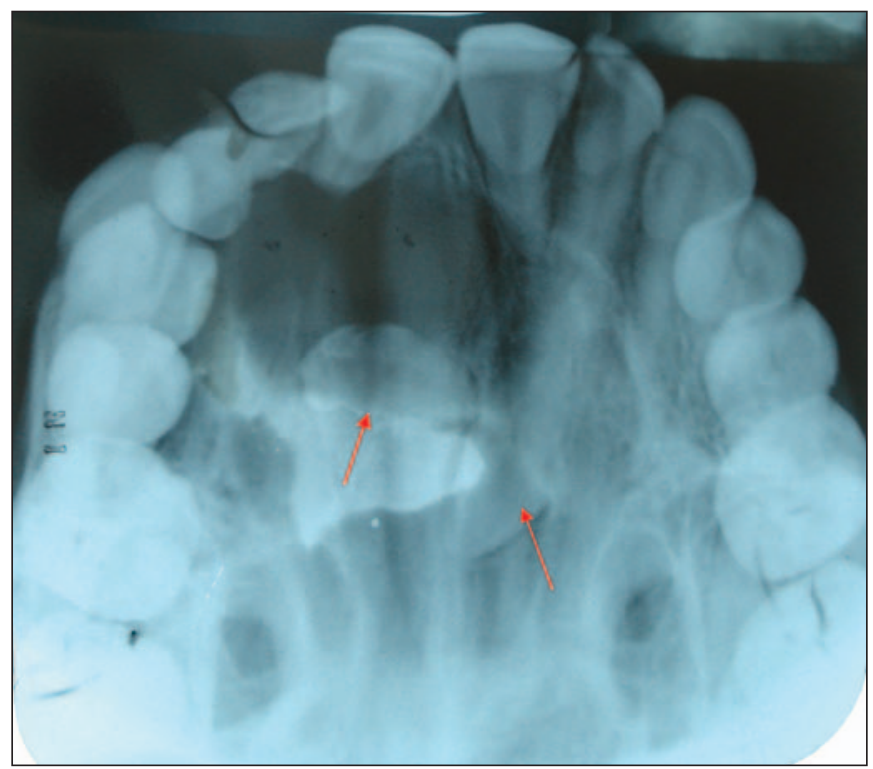

Fig. 5. Rx oclusal.
El informe radiológico fue: Lesión lítica a nivel de maxilar superior en su vertiente derecha de $2 \times 2 \mathrm{~cm}$ de tamaño aproximado, unilocular, de características expansivas, visualizando en su interior un diente incluido, que produce desplazamiento de los dientes adyacentes. No se acompaña de componente de partes blandas. Estos hallazgos están en relación con quiste dentígero (folicular) como primera posibilidad diagnostica. Además del diente incluido en el quiste y que presenta una localización superior a los incisivos de hemimaxilar derecho, se reobserva además la presencia en posición superior a incisivos de hemimaxilar izquierdo de un segundo diente supernumerario (mesiodens). (Figs 6-8)

Teniendo en cuenta su relación con un diente retenido, junto con el examen clínico y radiológico, se hizo un diagnostico presuntivo de quiste dentígero o folicular de tipo central o coronario. Dada su semejanza, con otras lesiones de apariencia quísticas, como otros quistes de origen odontogénico (quiste radicular, quiste periapical, queratoquiste odontogénico) y quistes de origen no odontogénicos (quiste del conducto incisivo, quiste nasolabial, Cavidad de Stafne, quiste aneurismático y ameloblastoma) se hizo diagnóstico diferencial con este tipo de patologías. $(1,5)$ La paciente fue derivada al Servicio de Cirugía Oral y Maxilo Facial del Hospital Clínico Universitario "Puerta del Mar" en Cádiz para su correspondiente tratamiento quirúrgico.

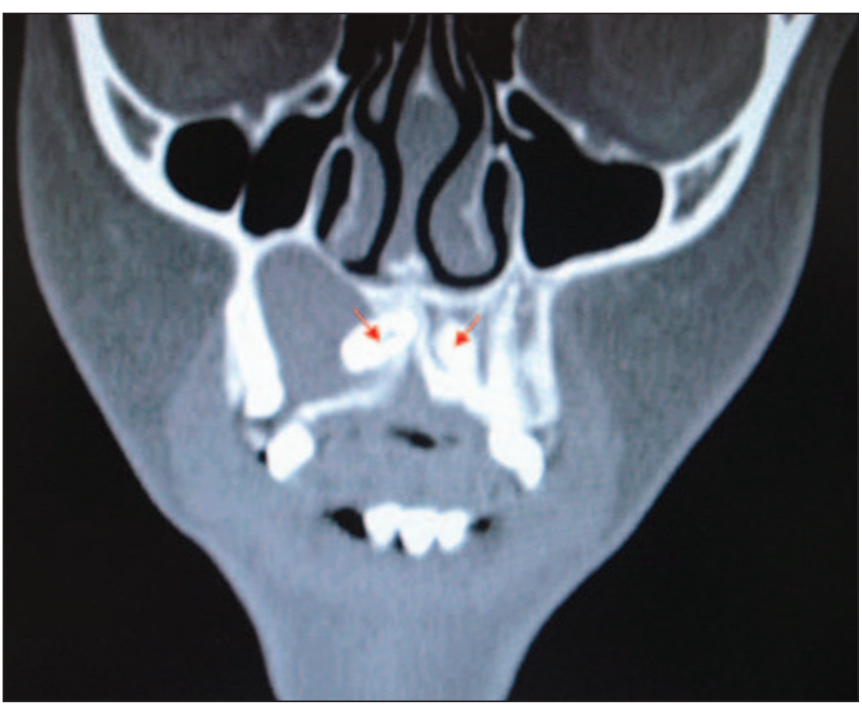

Fig. 6. T.A.C. 


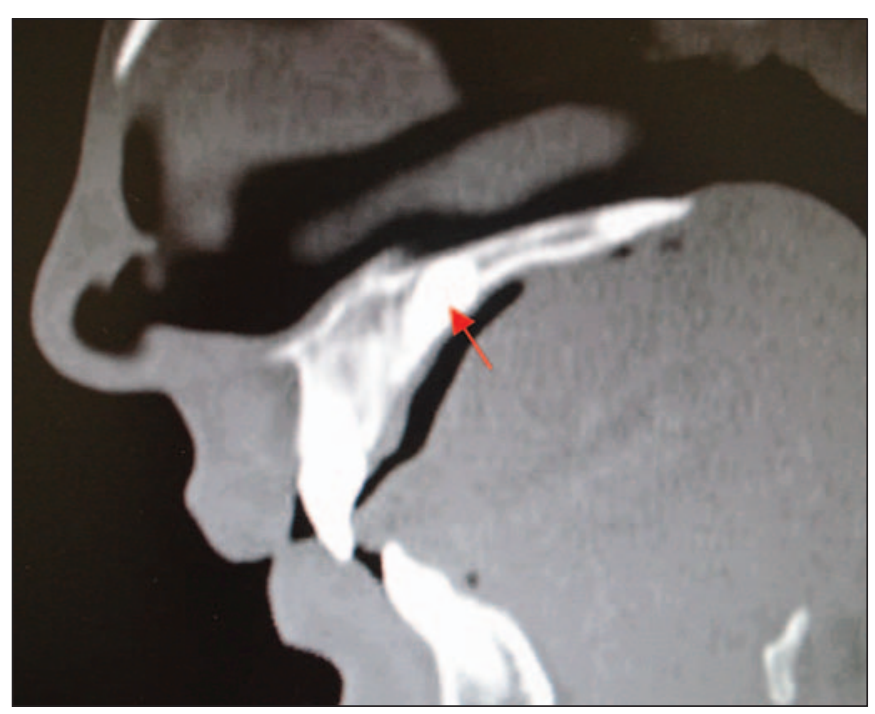

Fig. 7. T.A.C.

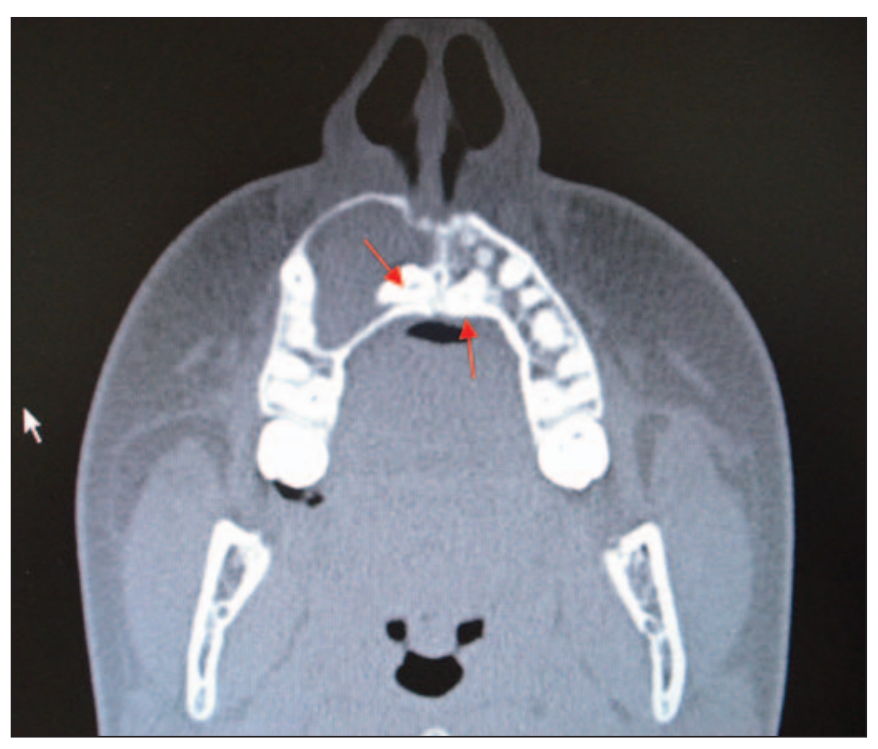

Fig. 8. T.A.C.

\section{ESTUDIO ANATOMOPATOLÓGICO}

Histológicamente, estos quistes están revestidos por un epitelio escamoso estratificado fino y regular, que se inserta en el límite cemento adamantino, caracterizado por la presencia de 2 a 4 capas de células cuboides o aplanadas que descansan en una fina lámina basal (Figs. 9-11). Este epitelio puede presentar engrosamientos y/o discontinuidades y entre sus células pueden aparecer células mucosas y cilia-

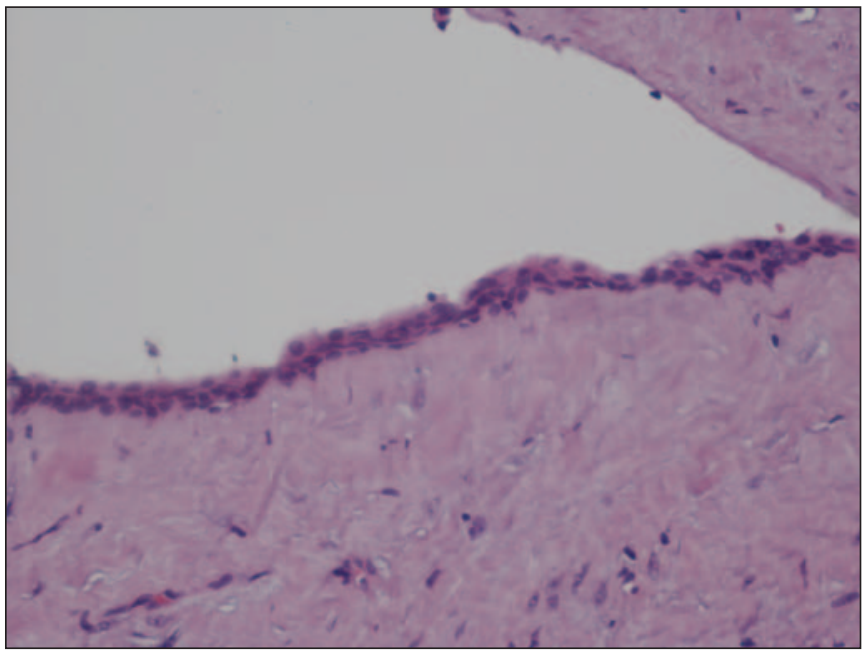

Fig. 9. $(\mathrm{HE} 20 \times)$.
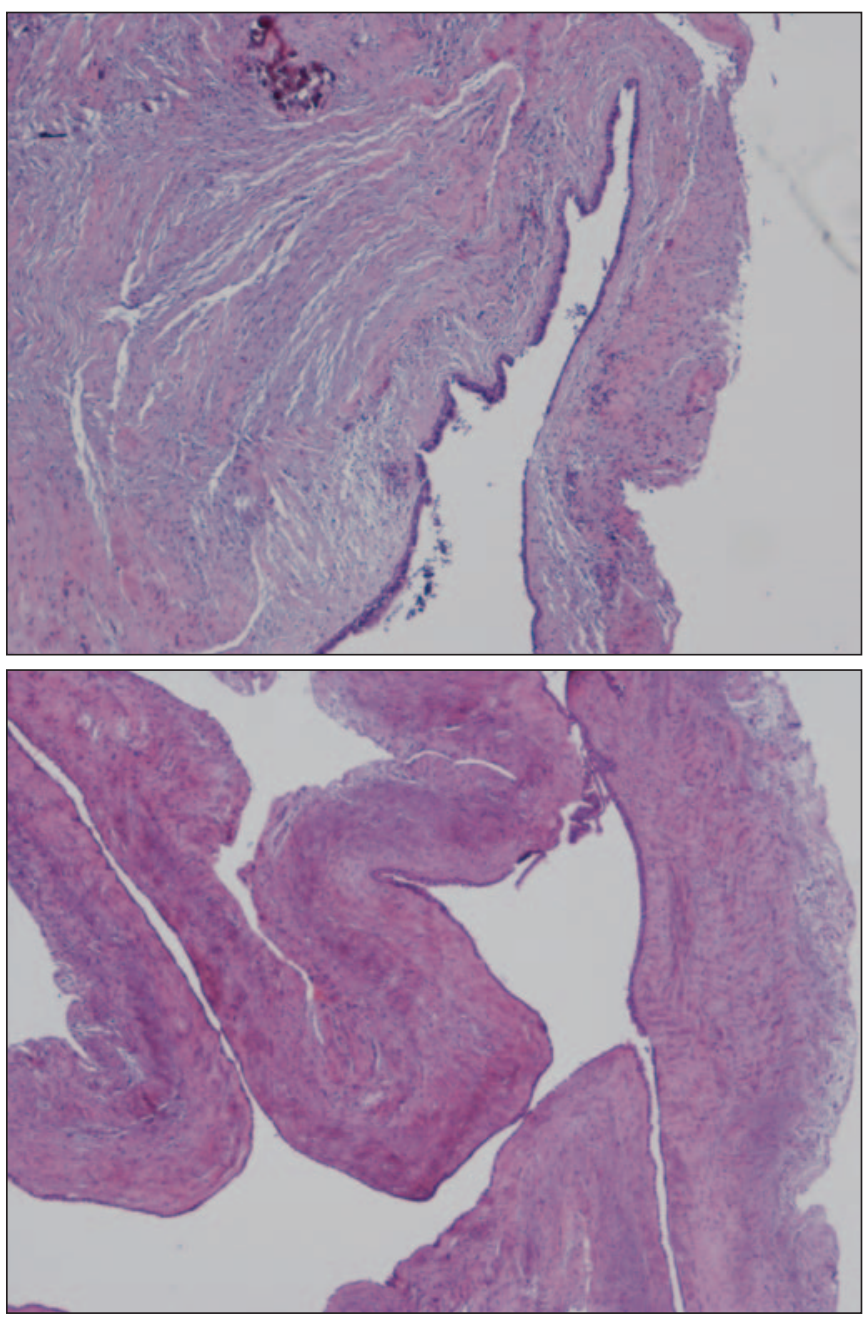

Figs. 10 y 11 (HE $4 \times-1$ y HE $4 \times-2)$. Fragmentos de pared del quiste, tapizado por epitelio simple y tejido conectivo denso subepitelial con poca celularidad fusiforme y presencia de calcificaciones dismórficas. 
das. Usualmente no están queratinizados, y la cápsula fibrosa que lo rodea presenta escaso o nulo infiltrado inflamatorio crónico. HE4x-3 No es raro encontrar en su interior cristales de colesterol, células gigantes multinucleadas y hemosiderina, motivo por el cual es difícil diferenciarlo (histológicamente) de un quiste radicular si ha habido infección (12, 15, 16) (Fig. 12).

\section{TRATAMIENTO}

Las lesiones uniloculares pequeñas se eliminan a través de una osteotomía, extracción del diente impactado y remoción de la lesión. En las lesiones grandes puede ser necesaria una cirugía extensa donde incluso puede haber resección de mandíbula. Hay dos procedimientos quirúrgicos que se emplean para eliminar estos quistes, marsupialización y enucleación. La marsupialización es un procedimiento indicado en aquellos casos donde se presentan quistes dentígeros de gran tamaño, que involucran una seria pérdida ósea y que adelgazan peligrosamente el hueso; es muy utilizado sobre todo en niños, en los casos que se pueda guiar la erupción del diente impactado hasta llevarlo a una posición normal (16). Con relación a la enucleación, siempre que sea posible se prefiere este procedimiento, porque pueden existir transformaciones ameloblásticas o carcinomatosas asociadas a la pared del quiste dentígero, lo

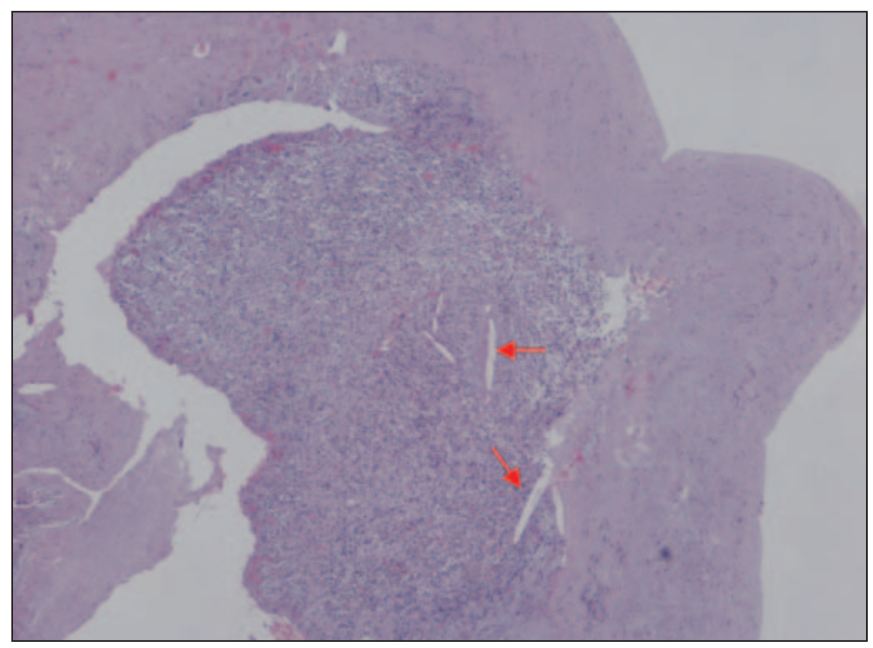

Fig. $12(\mathrm{HE} 4 \times-3)$. Infiltrado inflamatorio mixto con presencia de cristales de colesterol. cual hace necesario enuclear el quiste y someterlo a un estudio histopatológico, de esta manera se hace una escisión total del tejido patológico, disminuyendo la posibilidad de recurrencias y de transformaciones desfavorables (16). En el caso que se describe se realizó enucleación completa de las paredes del quiste, teniendo mucho énfasis en su eliminación completa, dada la posibilidad de transformación maligna. Se realizó, osteotomía por vestibular de maxilar superior, eliminando los dientes supernumerarios y la pared del quiste. El lecho quirúrgico se relleno con hidroxiapatita mas antibiótico, presentando como efecto secundario reacción inflamatoria, siendo tratada con corticoides. Después de 6 meses de seguimiento, realizamos una ortopantomografía de control, encontrando el canino definitivo 1.3 en unas condiciones fisiológicas normales, ausencia de anormalidades dentales y erupción en posición correcta en la cavidad oral (Fig. 13)

\section{DIAGNOSTICO DIFERENCIAL}

\section{A) Con quistes epiteliales}

1. Del desarrollo - Odontogénicos

- Queratoquiste o quiste primordial, derivan de la lámina dental indiferenciada y que no se asocia a una corona dental. Generalmente aparecen en mandíbula en región de ter-

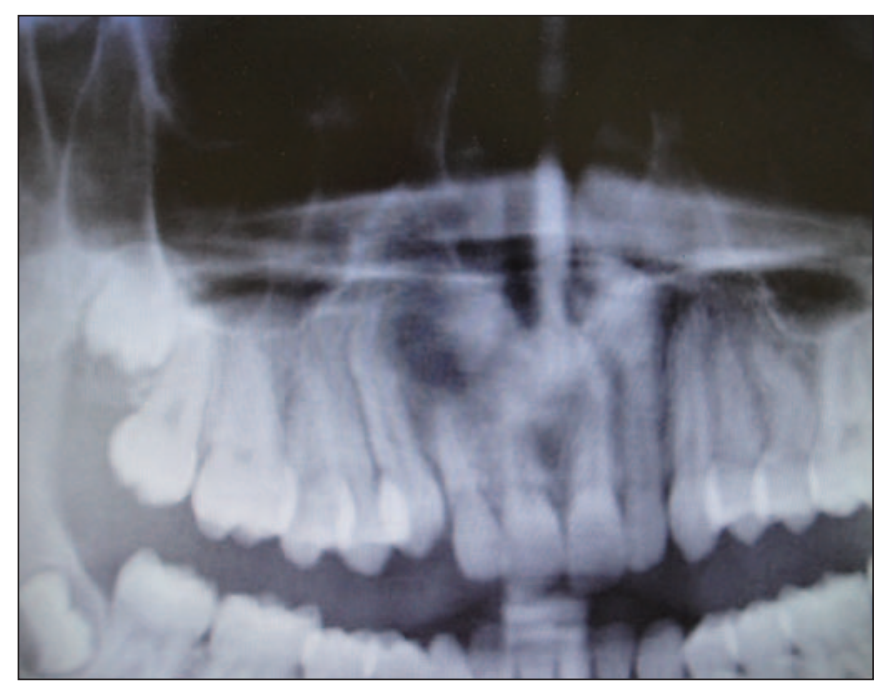

Fig. 13. 
ceros molares y es frecuente su extensión ascendente por la rama mandibular.

Radiológicamente se presentan como una lesión radiolúcida uní o multilocular, de bordes escleróticos lisos o dentados. Su característica principal es la ausencia de relación con dientes. Tiene gran tendencia a la recidiva. $(1,12,16,30)$

\section{- No odontogénicos}

Son observados en la región del canal incisivo o región nasolabial.

- El quiste nasopalatino se localiza en la línea media entre las raíces de los incisivos centrales maxilares. Aparecen como una imagen quística unilocular, y es característica su forma de corazón. Pueden causar divergencia en las raíces de los incisivos centrales, pero no contactan con los ápices dentarios. $(12,16,30)$

- Quiste nasolabial afecta a tejidos blandos del ala lateral de la nariz y labio superior. Son diagnosticados por su clásica localización anatómica. $(12,16,30)$

\section{Inflamatorios}

- Quiste radicular es el quiste más común de origen odontogénico en maxilar y mandíbula en personas adultas. $(12,29)$

Radiológicamente, están relacionados con la raíz de un diente cariado desvitalizado. $(12,16)$ Un gran quiste radicular puede producir infección y fistulización, crecimiento expansivo con deformación de tejidos, reabsorber la raíz de un diente adyacente, causar movilidad dentaria y extenderse al seno maxilar.

Su diagnóstico diferencial más frecuente, se hará con el granuloma periapical, no existiendo criterios fiables que permitan su diferenciación. Sus características son: su forma redondeada, su cortical bien delimitada, y un tamaño de unos $2 \mathrm{~cm}$. $(12,29)$

\section{B) No EPITELIALES}

- Quiste óseo simple, traumático o hemorrágico o de extravasación, es un quiste unilocular, que aparece generalmente en la región posterior del cuerpo mandibular entre caninos y tercer molar, siendo su segunda localización mas frecuente la sínfisis mandibular. Radiográficamente aparecen como una lesión ra- diolúcida bien delimitada con márgenes escleróticos. El margen del quiste óseo simple puede ser festoneado entre las raíces de los dientes afectados. $(12,16)$

- Cavidad ósea de Stafne, por lo general se localiza en el ángulo de la mandíbula. Su diagnostico es por casualidad, se presenta como una lesión radiolúcida única, bien definida, no expansiva y localizada bajo el conducto dentario inferior. $(12,16)$

- Quiste óseo aneurismático. La mayor parte de estos quistes, aparecen en otros huesos, solo el $2 \%$ aparecen en maxilar y mandíbula, afectando con mayor frecuencia a la mandíbula. Su forma de presentación es una lesión radiolúcida unilocular o multilocular en forma de panal de abeja. La TC/IRM puede revelar la presencia de sangre o el contenido liquido del quiste. (16)

\section{C) TUMORES}

- Ameloblastoma es una lesión radiolúcida uní o multilocular que causa expansión de la cortical, son mas frecuentes en la mandíbula (85\%) que en maxilar, de estas el $75 \%$ se localizan en la región molar ascendente. Presenta márgenes escleróticos dentados bien definidos, imagen en "pompa de jabón". Las raíces dentarias de los dientes vecinos se pueden desplazar o reabsorber. Cuando son uniloculares son indiferenciables del quiste folicular o dentígero. Tienen tendencia a invadir las trabéculas óseas y penetrar en la cortical mandibular. Muestra una elevada tasa de recidiva (55-90\%). (12, 16, 30)

\section{DISCUSIÓN}

Generalmente son asintomático, a no ser que exista una exacerbación inflamatoria aguda, semejándose estos casos a una celulitis o a una sinusitis (infección, dolor facial). (1, 10)

En mandíbula pueden provocar parestesias por afectación de nervio alveolar inferior. (11) Si son mayores de $2 \mathrm{~cm}$ de diámetro, los síntomas pueden ser: inflamación, provocar desplazamientos de dientes vecinos, movilidad de los dientes contiguos, reab- 
sorción radicular de dientes adyacentes y aumento de la sensibilidad dental. (11)

En mandíbula son los quistes de origen odontogénico mas frecuentes y representan aproximadamente el $20-24 \%$ de todos los quistes mandibulares, recubiertos de epitelio. (11)

Se debe hacer diagnostico diferencial con el saco folicular. Un ensanchamiento del espacio pericoronario que supere los $2,5 \mathrm{~mm}$ indicará la presencia de un quiste folicular en más del $75 \%$ de los casos, pudiendo medir éste hasta varios centímetros. $(1,12,13)$

Radiológicamente se manifiestan como una imagen radiolúcida, redondeada, bien delimitada, con bordes hiperostóticos, por tanto radiopacos, generalmente uniloculares. (12)

Ocasionalmente pueden aparecer lesiones múltiples (esto debe hacer sospechar la presencia del síndrome de nevus de células básales) o lesiones multiloculares (que debe diferenciarse del ameloblastoma). (12)

Incluyen en su interior la corona de un diente, dejando generalmente fuera, la raíz dentaria. (1) El diente relacionado generalmente se encuentra desplazado de su posición habitual (hacia apical). $(1,8,14,15)$

Las complicaciones asociadas que pueden provoca incluyen: fracturas óseas patológicas, pérdida de dientes permanentes, deformaciones óseas, pueden infectarse y desarrollar o transformarse en carcinoma de células escamosas, $(12,17)$ carcinoma mucoepidermoide y ameloblastoma (5\% de los quistes foliculares). (12, 18)

La presencia de inflamación en el labio superior, puede ser el resultado, de un tumor salival, infección regional o la presencia de un quiste dentígero. $(7,19)$ En nuestro caso la causa fue la presencia de un quiste dentígero.

Existen pocos estudios sobre patología oral en pacientes pediátricos. Tyrologou S. y col (20) en un estudio con 11.500 niños, estudio los rasgos clínicos y radiológicos de 123 mesiodens. No encontró patología quística asociada a éstos, ni reabsorciones radiculares en dientes adyacentes, llegando a la con- clusión, que el riesgo de complicaciones causadas por mesiodens, parece ser muy bajo, recomendando una actitud más restrictiva en su extracción eliminación quirúrgica.

Igualmente, Gunduz y col. (21) estudiaron los rasgos radiológicos y la frecuencia de mesiodens en una población de 23.000 niños, de los cuales 69 presentaron 85 casos de mesiodens, 47 eran niños y 22 niñas por tanto, la proporción fue de 2,1:1. Las complicaciones asociadas a estos fueron erupción retrasada de los incisivos permanentes en un $38,8 \%$, diastemas en un $17,6 \%$, rotación axial o inclinación de incisivos erupcionados permanentes en un 16,4\% y reabsorción de los dientes permanentes en un $4,7 \%$. La prevalencia de los mesiodens fue del 0,15 al 2,2 \%. Ningún caso de quiste dentígero asociado fue descrito.

Sin embargo, Asaumi y col. (22) diagnosticaron la presencia de 256 casos de mesiodens en un total de 200 pacientes. Entre las complicaciones describieron la presencia de 22 casos de quistes dentígeros (11\%). Estos autores llegaron a la conclusión, de que la formación de quistes dentígeros por un mesiodens, puede estar influenciada por periodos largos de impactación.

Se considera que constituyen mas del $24 \%$ de los quistes de mandíbula. La incidencia de desarrollo de quistes dentígeros que afecten a supernumerario es del $1,5 \%$, igual que para los incisivos centrales maxilares, comparado con el $45,7 \%$ que implican a terceros molares mandibulares. (23) Para Mourshed la probabilidad de que un diente incluidos desarrolle un quiste dentígero es del 1,44\%. (24) Para Daley y col. la probabilidad es del 0,1-0,6\% (25) y para Shear (23) es del $1.5 \%$.

Cuando aparecen múltiples, suelen asociarse a condiciones como: mucopolisacaridosis y síndrome de nevo de células basales. (26)

Generalmente aparecen en la $2^{\mathrm{a}}$ y $3^{\mathrm{a}}$ década de la vida. (1) Pueden afectar a niños (9) y adolescentes (14) y tienen una leve predilección por el sexo masculino 2:1. $(1,2,15)$ En este caso presentamos, un quiste dentígero asociado a un diente supernumerario en una adolescente de 14 años. 
Pina Godoy y col (27) realizaron una evaluación epidemiológica en 100 pacientes de 0 a 14 años de edad con diagnóstico histopatológico de quiste odontogénicos. La edad media fue de 9,78 años con una ligera predilección por el sexo masculino. La región posterior mandibular y anterior maxilar fueron las más afectadas. El quiste dentígero fue la lesión más común, seguida por el quiste radicular. Este estudio demuestra que en niños los quiste de origen odontogénicos más frecuentes, son los quistes dentígeros.

Igualmente Ochsenius y col, en un estudio, en paciente igual o menores de 15 años, los quistes de desarrollo odontogénicos, como el quiste dentígero, fueron mas frecuentes que los quistes inflamatorios. (28)

Sousa y col. (31) en el año 2002 publicaron los resultados de 2.356 biopsias, estas las dividieron en 20 grupos, no existiendo diferencias significativas por sexo. La patología mas prevalente fue el mucocele con un $13,5 \%$ de los casos, seguido por los quistes dentígeros con un 6,5\% y la hiperplasia fibrosa con un $5,4 \%$. Los odontomas y ameloblastoma tuvieron una incidencia significativa.

Recientemente Shah SK y col. (32), publican una revisión de 5.457 biopsias en paciente entre 0 y 16 años de edad. Las lesiones las dividieron en a) Lesiones del metabolismo, b) Inflamatorias, c) Neoplásicas y 4) del Desarrollo. El resultado fue que las lesiones inflamatorias constituyeron el mayor $\mathrm{n}^{\circ}$ de casos, con un $51 \%$, seguido por las lesiones del desarrollo, con un 35\%, y las tumorales, con un $13 \%$. Los quistes dentígeros fueron las lesiones mas frecuentes, seguido por los quistes mucosos.

Avelar EL y col. (33), determinan la prevalencia de quistes de desarrollo odontogénico y los comparó con otros estudios internacionales. De todas las lesiones biopsiadas los quistes odontogénicos representaron un 9,94\%. La media de edad fue de 28,9 años y el $57,6 \%$ el sexo fue masculino. En este estudio, no se hace mención de edad de los pacientes, por lo que se supone que los pacientes estudiados fueron de todas las edades. Los quistes radiculares fueron la lesión mas prevalente con un $52,2 \%$ de los casos. La mandíbula fue el lugar donde más frecuente se presentaron las lesiones con un
$56 \%$ de los casos. Este estudio concuerda con otros autores.

En resumen, los quistes odontogénicos como el quiste dentígero es la lesión mas frecuente junto con el mucocele en edad pediátrica, no existiendo diferencias con respecto a la edad.

En personas adultas o en la población general el quiste radicular es el más frecuente, existiendo un ligero predominio en hombres. La región mas afectada es la mandíbula.

\section{CONCLUSIONES}

En nuestro caso, presentamos un inusual caso de dos supernumerarios mesiodens retenidos, en una joven de 14 años edad, uno de ellos, asociado a un quiste dentígeno y destacamos los diagnósticos diferenciales más relevantes con otras patologías quísticas semejantes.

En edad pediátrica los quistes dentígeros junto con los mucoceles son las lesiones orales mas frecuentes, con respecto a la edad difieren según el autor

Es importante realizar un diagnóstico temprano de este tipo de quiste debido a la posibilidad de presentar complicaciones como reabsorción de las piezas adyacentes, maloclusiones y su posible transformación maligna.

Los dientes supernumerarios por lo general en niños y adolescentes pueden provocar problemas ortodónticos. En este caso la paciente solo presentaba, retención en arcada del canino deciduo 5.3, como consecuencia de la impactación del canino permanente 1.3.

Son los quistes de origen odontogénico mas frecuentes en personas jóvenes, con una ligera predilección por el sexo masculino.

El tratamiento a realizar en este tipo de quiste, depende del tamaño y la relación de este con otras estructuras.

Dada la capacidad de producir complicaciones, el diagnóstico debe ser lo mas precoz posible. 
Es necesario concienciar a la población de la necesidad de someterse a controles odontológicos periódicos.

El estudio anatomopatológico es imprescindible, así como el control de los pacientes a corto, mediano y largo plazo

En edad pediátrica los quistes dentígeros junto con los mucoceles son las lesiones mas frecuentes, con respecto a la edad difieren según el autor.

\section{BIBLIOGRAFÍA}

1. Bascones Martínez A. y col. Tratado de Odontología Volumen II. Trigo Ediciones S.L. Madrid Primera Edición: Octubre 1998.

2. Ikeshima A, Tamura Y. Differential diagnosis between dentigerous cyst and benign tumor with an embedded tooth. J Oral Sci 2002;44: 13-7.

3. Gondin JO, Neto JJ, Nogueira RL, Giro EM. Consevative Management of a dentigerous cyst secondary to primary tooth trauma. Dent Traumatol. 2008 Dec;24(6):676-9.

4. Sannomiya EK, Nogueira Mde O, Diniz M de C, Pacca FO, Dalben Gda S. Trauma-induced. dentigerous cyst involving the anterior maxilla. J Dent Child (Chic). 2007 May-Aug;74(2):1614.

5. Dinkar AD, Dawasaz AA, Shenoy S. Dentigerous cyst associated with multiples mesiodens: A case report. J Indian Soc Pedod Prev Dent. 2007 Mar; 25(1):56-9.

6. Lustmann J, Bodner L. Dentigerous cysts associated with supernumerary teeth. Int $\mathrm{J}$ Oral Maxillofac Surg 1988;17:100-102.

7. Khan MH, Alam MT, Haque S, Khan SH, Fatema CN, Yahsin T,Choudhury AR. Upper lip swelling caused by a large dentigerous cyst with mesiodens Mymensingh Med J. 2008 Jul;17(2 Suppl):S100-3.
8. Ziccardi VB, Eggleston TE, Schnider RE. Using fenestration technique to treat a large dentigerous cyst. J Am Dent Assoc 1997;128:201-5.

9. Passi S, Gauba K, Agnihotri A, Sharma R. Dentigerous cyst in primary dentition: A case report J Indian Soc of Pedod Prev Dent. 2008 Dec;26(4): 168-70.

10. Manzzor T, Raza SN, Oayyum A, Azam K. Dentigerous cyst presenting as facial pain. J Coll Physicinas Surg Pak. 2006 Dec;16(12):783-5.

11. Sumer M, Bap B, Yýldýz L. Inferior alveolar nerve paresthesia caused by a dentigerous cyst associated with three teeth. Med Oral Patol Oral Cir Bucal 2007;12:E388-90.

12. Raspall G. Enfermedades maxilares y craneofaciales. Atlas Clinico. 1990 Salvat Editores, S.A.

13. Bodner L, Woldenberg Y, Bar-Ziv J. Radiographic features of large cysts lesion of jaws in children. Pediatr Radiol 2003;33:3-6.

14. Kalaskar RR, Tiku A, Damle SG. Dentigerous cysts of anterior maxilla in a young child: A case report. J Indian Soc Pedod Prev Dent. 2007 OctDec;25(4):187-90.

15. Bagan JV, Scully C. Medicina y Patología Oral. Ed. Medicina Oral S.L. 2.006.

16. Laskin D. Oral and Maxillofacial Surgery. Editorial Medica Panamericana 1987

17. Gulbranson SH, Wolfrey JD, Raines JM, McNally BP. Squamous cell carcinoma arising in a dentigerous cyst in a 16-month-old girl. Otolaryngol Head Neck Surg. 2002 Nov; 127 (5):463-4.

18. Chakraborty A, Sarkar S, Dutta BB. Localized disturbances associated with primary teeth eruption. J Indian Soc Pedod Prev Dent 1994; 12:25-8.

19. Scolozzi P, Lombardi T, Richter M. Upper lip swelling caused by a large dentigerous cyst. Eur Arch Otorhinolaryngol 2004; 5: (E pub ahead of print). 
20. Yyrologous S, Koch G, Kurol J. Location, complications and treatment of mesiodentes-a retrospective study in children. Swed Dent J. 2005;29(1):1-9.

21. Günduz K, Celenk P, Zengin Z, Sümer P. Mesiodens: a radiographic study in children. Journal of Oral Science. 2008 Sep;50(3):287-91.

22. Asaumi JI, Shibata Y, Yanagi Y, Hisatomi M, Matsuzaki $\mathrm{H}$, Konouchi $\mathrm{H}$, et al. Radiographic examination of mesiodens and their associated complications. Dentomaxillofac Radiol 2004;33: 125-7.

23. Shear M. Dentigerous cyst of oral region. $2^{\text {nd }}$ ed. Wright PSG: Bristol; 1983. pp. 56-75

24. Mourshed F. A roentgenographic study of dentigerous cysts, II: Role of roentgenograms in detecting dentigerous cyst in the early stages. Oral Surg 1964;18:54-61.

25. Daley TD, Wysock GP. The small dentigerous cyst, the diagnostic dilemma. Oral Surg Oral Med Oral Pathol Oral Radiol Endod 1995;79:77-81.

26. Ustuner E, Fitoz S, Atasoy C, Erden I, Akyar S. Billateral maxillary dentigerous cyst: A case report. Oral Surg Oral Med Oral Pathol Oral Radiol Endod. 2003 may;95(5):632-5.

27. Pina Godoy G, Dantas da Silveira, É J, GordónNuñez M A et al. Quistes de los maxilares en niños: un análisis clínico. Acta Odontol. Venez, dic. 2007, vol.45, no.4, p.546-549.
28. Ochsenius G, Escobar E, Godoy L, Peñafiel C. Odontogenic cyst: analysis of 2.944 cases in Chile. Med Oral Patol Oral Cir Bucal. 2007 Mar 1;12(2):E85-91.

29. Mosqueda A, Irigoyen ME, Díaz MA, Torres MA. Quistes odontogénicos. Análisis de 856 casos. Medicina Oral 2002; 7: 89-96.

30. Grover SB, Singh P, Venkatachalam VP, Hekha N. Mesiodens presenting as a dentigerous cyst: Case Report. J Radiol Imaging 2005;15:69-72.

31. Sousa FB, Etges A, Corréa L, Mesquita RA, de Araujo NS, Pediatric oral lesions: a 15-years review from Sâo Paulo, Brasil. J Clin Pediatric Dent Summer; 26(4):413-8.

32. Shah SK, Le MC, Carpenter MM, Retrospective review of pediatric oral lesions from a dental school biopsy service. Pediatric Dent, 2.009 JanFeb;31(1):14-9.

33. Avelar RL, Antunes AA, Carvalho RW, Becerrra PG, Oliveira Neto PJ, Andrade ES, Odontogenic cysts: a clinicopathological study of 507 cases. J Oral Sci. 2009 Dec;5(4):581-6.

\section{CORRESPONDENCIA}

Fco. Javier Rodríguez Romero

Centro de Salud "La Paz"

Jerónimo de la Concepción, s/n

Cádiz

E-mail: frrodrom@gmail.com 